\title{
The intense magnetic storm of March 1918 as recorded by observatory Ógyalla (present day Hurbanovo)
}

\author{
Fridrich Valach ${ }^{1}$ (D)
}

Received: 24 March 2016/ Accepted: 28 June 2016/Published online: 13 July 2016

(C) Akadémiai Kiadó 2016

\begin{abstract}
This paper presents an analysis of the magnetic storm on March 8, 1918, on the basis of a preserved analogue magnetogram that was recorded by observatory Ógyalla, present day Hurbanovo. It is inferred that much of the spectacular phenomena that were attributed to this storm, such as effects in telegraph lines, were likely caused by rapid changes of the geomagnetic field during two consecutive substorms. The storm sudden commencement that preceded the magnetic storm was probably also potent in this regard. This supports the recent findings that argue against the inevitable major role of the ring current in extreme magnetic disturbances at mid latitudes.
\end{abstract}

Keywords Magnetic storm · Magnetic observatory · Magnetogram · History

\section{Introduction}

In recent years much research has focused on space weather, representing a challenging scientific field, where large amount of data is needed. High-quality spaceborn data are abundantly available from different space missions and fleets of probes. However, some observations of phenomena that are related to space weather commenced long time before the era of the space probes. An example of this is the monitoring of the geomagnetic field by a few classical magnetic observatories in olden times. Of particular concern are the records of the most extreme historical magnetic storms. Since the extreme storms are rare phenomena, the historical records of them are surely very valuable for the researchers dealing with space weather.

Fridrich Valach

fridrich@geomag.sk; fred.valach@gmail.com

1 Geomagnetic Observatory, Earth Science Institute, Slovak Academy of Sciences, Komárňanská 108, 94701 Hurbanovo, Slovakia 
The Hurbanovo Geomagnetic Observatory of the Earth Science Institute SAS started its operation in 1893, in the era of the Austro-Hungarian Empire (Prigancová and Vörös 2001; Ochabová and Ochaba 1977). The archive of the observatory preserves a collection of precious magnetograms that were recorded during years 1903-1918, when the observatory was known as Ógyalla Royal Hungarian ${ }^{1}$ Observatory for Meteorology and Geomagnetism. ${ }^{2}$ Unfortunately, not all of the records of that time were preserved-for instance, the magnetogram of interesting event of November 1, 1903, is missing.

Luckily, some other unique events also occurred within this period. One of them, the intense magnetic storm that occurred on March 8, 1918, is studied here. Focusing on this historical geomagnetic event, the contemporary historical name of the observatory, Ógyalla, is consistently utilized throughout this paper.

The event of March 1918 was indeed remarkable and the general public also took notice about it. For instance, The New York Times wrote about the aurora borealis in connection with a night attack of German aeroplanes to London:

'LONDON, March 8.- Seven or eight German aeroplanes made a raid over England last night. Two of them reached London and dropped bombs in the north, northeast, and southwest districts of the city. [...] It was the first time the enemy had attempted a night raid over London when there was no moon. [...] There was a remarkable display of the aurora borealis last night, and it is believed by many that this furnished conditions under which the air raiders could work more effectively than under a clear, star-lit sky. Watchers on the Kent coast said that just before they heard the raiders approaching [,] the whole northern sky became illuminated, in bands of red and white light which shone over the sea with far more powerful effect than the full moon.' (Quoted from The New York Times, March 9, 1918, page 3).

There were also many other reports on the aurora and on affecting the telegraph lines by the peculiar magnetic disturbance in contemporary newspapers. They were collected for example by Odenwald (2016). The effects were observed in Europe as well as in America.

When studying historical geomagnetic events, the problem often arise from the lack of direct observations. For example, the exploration of the famous Carrington superstorm, that occurred on September 2, 1859, relies primarily on the series of horizontal intensity ${ }^{3}$ observed at Colaba, India (e.g. Tsurutani et al. 2003; Cliver and Dietrich 2013).

Recently, Cid et al. (2015) investigated an event which was similar to the Carrington event. This 'Carrington-like' event was the magnetic storm recorded by the Tihany observatory, Hungary, on October 29, 2003. The authors showed that the immensely large decrease of horizontal intensity that was observed at Colaba was caused by the fieldaligned currents (FACs). This finding contradicted the traditional opinion, in which the intensified ring current was thought to be the main cause of that large decrease.

Additionally, it was suggested by Cid et al. (2015) that magnetic storms similar to the Carrington event might occur more commonly than it was thought until now. One can argue that the 'Carrington-like' events do not manifest the violent decrease of the horizontal intensity everywhere on the globe. On the contrary, such a violent decrease can just occur as a local feature.

\footnotetext{
1 The term Royal Hungarian marked the governmental bodies of the Kingdom of Hungary in the frame of Austro-Hungarian Empire.

2 The full original name of this observatory was Ógyalla m. kir. Meteorológiai és Földmágnességi Obszervatoriuma.

${ }^{3}$ For the sake of conciseness, the term 'horizontal intensity' is utilised throughout this paper for the horizontal component of the geomagnetic field.
} 
Subsequently, Saiz et al. (2016) reviewed the records of the geomagnetic field from nine observatories in the period from 1991 to 2009 and they discovered another 'Carrington-like' event. This was the magnetic storm that was launched on January 21, 2005. Saiz and her co-workers found the resemblance between the record of the Carrington storm at Colaba and the record of the 'Carrington-like' event at the magnetic observatory of the Stennis Space Center, BSL, in the United States of America.

The purpose of this study is to point out that the intense magnetic storm on March 8, 1918, might have some features of a 'Carrington-like' event. An additional intention of this paper is to draw attention to the data that were observed by the Ógyalla observatory during the intriguing early part of this storm. These are the values of the horizontal intensity of the magnetic field, which we believe they should be interesting for researchers dealing with space weather and its manifestations on the surface of the Earth.

This paper is organised as follows: The magnetogram made at Ógyalla is presented in the next section, where also data of some other magnetic observatories are reviewed for comparison. Section 3 analyses and discusses the presented data. A conclusion is provided in Sect. 4.

\section{Description of materials}

In this section the magnetogram of the event in question together with a piece of information about the registering instruments are described first. Then the data of some other observatories that were working during the time of the studied storm are introduced.

\subsection{The record of the magnetic storm of March 1918 at Ógyalla}

During the time period of interest, two Mascart's type magnetographs made by Carpentier, were installed in the Ógyalla observatory. One of them was tailored to photographic recording. For the absolute measurements of magnetic declination and horizontal intensity the Wild theodolite was used. In 1918, the observatory did not perform the absolute measurements for the vertical component of the geomagnetic field as they found the vertical variometer unreliable; this state persisted since 1911 (Prigancová and Vörös 2001; Ochabová and Ochaba 1977).

The magnetogram recorded with the Mascart type magnetogpraph is shown in Fig. 1. Here the record catches the essential part of the magnetic storm on March 8, starting with the storm sudden commencement (ssc) that occurred at the end of March 7. The onset of the ssc on the magnetogram coincide with the ssc reported for 21:06 UT by the International Service on Rapid Magnetic Variations. ${ }^{4}$ The curves in the magnetogram are for horizontal intensity, magnetic declination and the vertical component of the geomagnetic field. The indicated time is the Greenwich Mean Time, which equals the Universal Time (UT). The baseline values for horizontal intensity and declination are $20898 \mathrm{nT}$ and $5^{\circ} 16.2^{\prime}$, respectively. The baseline value for the vertical component was not determined.

Information about the values of the geomagnetic field, which are presented in Sect. 3, was determined on the basis of numbers that were inscribed on the original magnetogram by observers in the past. Sensitivity of the instrument was estimated on the base of these

\footnotetext{
${ }^{4}$ http://www.obsebre.es/php/geomagnetisme/vrapides/ssc_1918_d.html.
} 


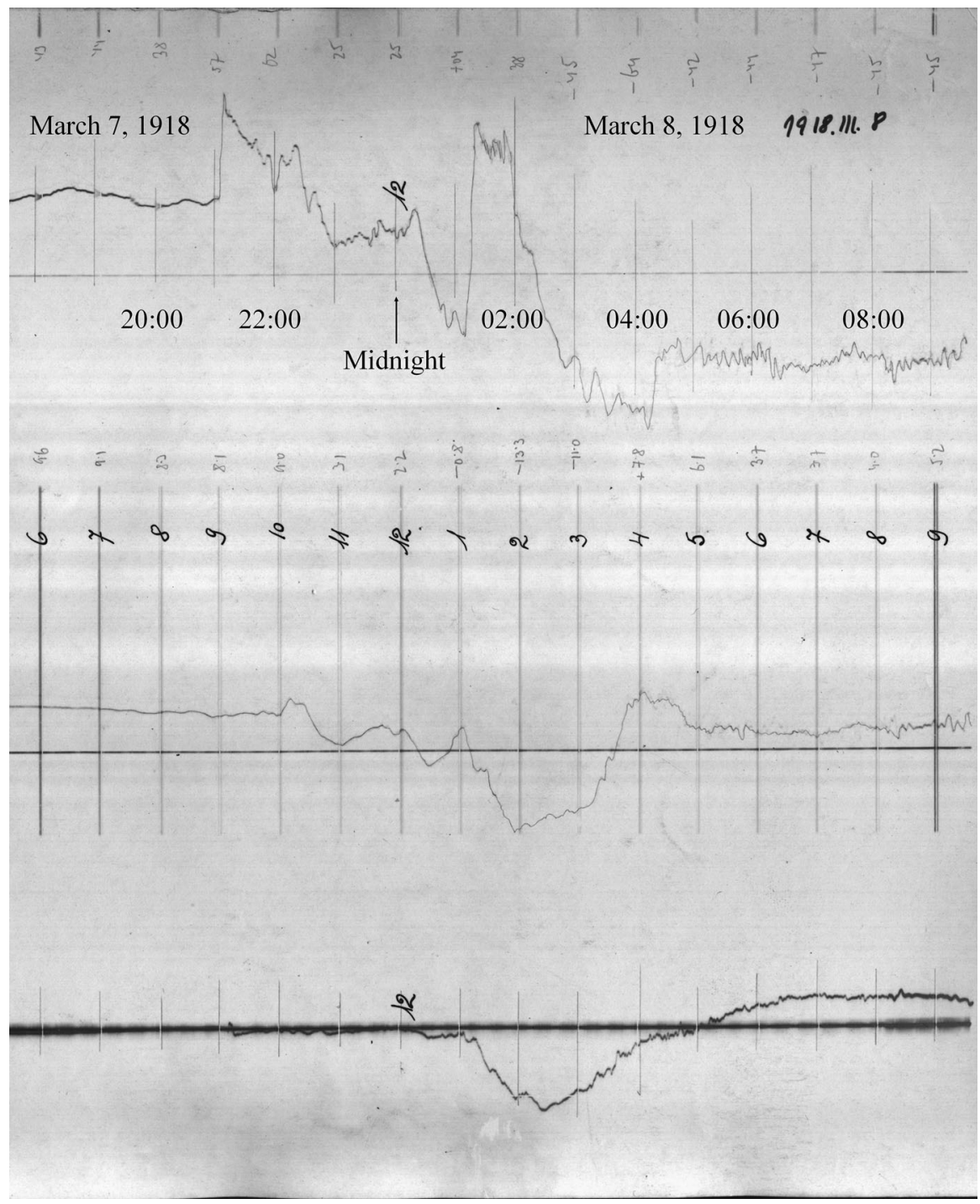

Fig. 1 The magnetogram that catches the onset as well as the main part of the magnetic storm from March 8,1918 , recorded at observatory Ógyalla. The curve at the top shows horizontal intensity, the middle curve corresponds to magnetic declination, and the bottom curve displays the vertical component of the geomagnetic field. The indicated time is the Greenwich Mean Time. Baseline values for horizontal intensity and declination are $20,898 \mathrm{nT}$ and $5^{\circ} 16.2^{\prime}$, respectively

numbers to be $3 \mathrm{nT}$ per millimetre and 1.3 arcminute per millimetre, respectively. In addition, values of horizontal intensity with 5-min sampling interval were determined by hand. Subsequently, these 5-min data were used for calculating 1-h means, which were then compared with 1-h means from other observatories. 


\subsection{Data from other observatories}

Analogue magnetograms from British observatories Eskdalemuir, Greenwich, Kew and Stornyhurst were also consulted. They are collected on the webpage of the British Geological Survey. 5

Besides the traditional analogue magnetograms, 1-h values of the horizontal intensity were analysed. The list of observatories from which data are considered here are shown in Table 1. The data were taken from the website of the World Data Center for Geomagnetism, ${ }^{6}$ Kyoto, which is operated by the Data Analysis Center for Geomagnetism and Space Magnetism, Graduate School of Science, Kyoto University, Japan.

\section{Data analysis and discussion}

In this section the event of March 1918 available on the magnetogram at Ógyalla is analysed and compared with magnetograms and 1-h data from some other magnetic observatories, listed in Table 1. The magnetic storm commenced with an ssc at 21:06 UT. During that initial event the horizontal intensity sharply increased by $52 \mathrm{nT}$. The duration of this event is estimated to be $4.8 \mathrm{~min}$. The International Service on Rapid Magnetic Variations reports that the duration of this ssc was $4.4 \mathrm{~min}$.

The main phase of the storm, which is the well-known result of the strengthening of the ring current, fully developed after 02:00 UT on March 8. Before that time, however, there was a rapid fall of the horizontal intensity and then immediately the magnetic field rose sharply to high values. The fall was by $58 \mathrm{nT}$ and it lasted $35 \mathrm{~min}$, from 00:18 to 00:53 UT on March 8. Between 00:53 and 01:07 UT a small peak appeared. The mentioned sharp rise then started at 01:07 UT and it lasted 14.5 min, reaching $107 \mathrm{nT}$.

The sharp rise looks like another ssc, but the International Service on Rapid Magnetic Variations does not report such an event on March 8. It seems that it was just the end of a magnetospheric (auroral) substorm. Additionally, the magnetograms made by the British observatories indicate that this was not a sole substorm - there were two substorms which occurred one after another. Especially the magnetograms of Greenwich and Kew indicate this clearly. At Ógyalla these two substorms overlapped and superimposed to one strong event; only the small peak between 00:53 UT and 01:07 UT separated them on the magnetogram.

The assumption that the disturbance between 00:18:00 and 01:21:30 UT was related to substorms rather than to the ring current can be supported by the following fact: this disturbance was not observed everywhere around the globe. The Figs. 2 and 3 demonstrate the presence or absence of this event for several magnetic observatories from Table 1. These are the observatories with horizontal intensity data available with 1-h time resolution from the webpage of the World Data Center for Geomagnetism, Kyoto (Sect. 2.2).

Despite that the 1-h sampling rate it relatively sparse, at least some traces of this event are expected to be visible in the figures. If the disturbance was caused by the ring current, the phenomena should be observed at all observatories. But it was observed only at European observatories De Bilt, Seddin, Val Joyeux, and Ógyalla (Fig. 2). On the other

\footnotetext{
5 http://www.bgs.ac.uk/data/Magnetograms/search.cfc?[continuedonthenextline]method=getResults\&from Month=3\&from Year=1918\&submitBtn=Submit+Query.

${ }^{6}$ http://wdc.kugi.kyoto-u.ac.jp/catmap/year.html.
} 
Table 1 Basic information about the geomagnetic observatories of which 1-h data were used in this study

\begin{tabular}{llccccc}
\hline Observatory name & IAGA code & \multicolumn{2}{c}{ Geographic coordinates } & & \multicolumn{2}{c}{ Geomagnetic coordinates } \\
& & Latitude & Longitude & & Latitude & Longitude \\
\hline Cheltenham & CLH & 38.70 & 283.20 & & 49.11 & 353.76 \\
De Bilt & DBN & 52.10 & 5.18 & & 53.28 & 90.46 \\
Eskdalemuir & ESK & 55.32 & 356.80 & & 57.86 & 83.85 \\
Honolulu & HON & 21.32 & 202.00 & & 21.60 & 269.45 \\
Ógyalla & OGY & 47.88 & 18.19 & & 46.67 & 101.18 \\
Seddin & SED & 52.28 & 13.01 & & 51.08 & 98.04 \\
Sitka & SIT & 57.06 & 224.67 & & 60.34 & 279.78 \\
Tucson & TUC & 32.17 & 249.27 & & 39.99 & 315.75 \\
Val Joyeux & VLJ & 48.82 & 2.01 & & 50.71 & 85.80 \\
Vassouras & VSS & -22.40 & 316.35 & & -13.09 & 26.39 \\
Vieques & VQS & 18.15 & 294.55 & & 28.54 & 6.62 \\
\hline
\end{tabular}

hand, the 1-h data of observatories Honolulu in Hawaii, Tucson in Arizona, and Vieques in Puerto Rico did not indicate any distinctive traces of substorms (Fig. 3).

There is also a temporary decrease of the horizontal intensity at 01:00 UT at Cheltenham, which is the observatory located on the East Coast of the United States. This time slightly differs from the time when similar disturbance occurred in Europe. Maybe this difference is caused by the routine that was used for calculation of 1-h values at the observatory. Another possible explanation is that this decrease was not related to the disturbance that was observed in Europe.

The set of data from Eskdalemuir located in Scotland, the United Kingdom, contains a gap during the key part of the magnetic storm. Nonetheless, the existing data (the data not shown here) do not contradict the above mentioned inference. On the other hand, the 1-h data of Sitka, which is located at high latitudes, as well as Vassouras on the Southern hemisphere showed a very different course of evolution (the data not shown). This is not a surprise because of their remote magnetic latitudes: $60.34^{\circ}$ for Sitka and $-13.09^{\circ}$ for Vassouras. Thus their data neither supports nor contradicts the above mentioned inference.

In summary, the disturbance between 00:18:00 and 01:21:30 UT appears to be a local phenomena that was likely caused by electric currents flowing in the ionosphere at high geomagnetic latitudes. The observed aurora borealis also indicates that the significant part of the sources of the geomagnetic disturbances that took place on March 8, 1918, were associated with the processes at high latitudes.

Information about variation of magnetic declination might be interesting, too. Figure 1 shows that the declination did not change significantly during the substorms. But after the substorms ceased, the declination decreased by 21 arcminute in a half-hour. During the next one hour declination increased by 30 arcminute. This variation of declination as well as the variation of the vertical component of the geomagnetic field were obviously caused by the strengthening of the ring current. 

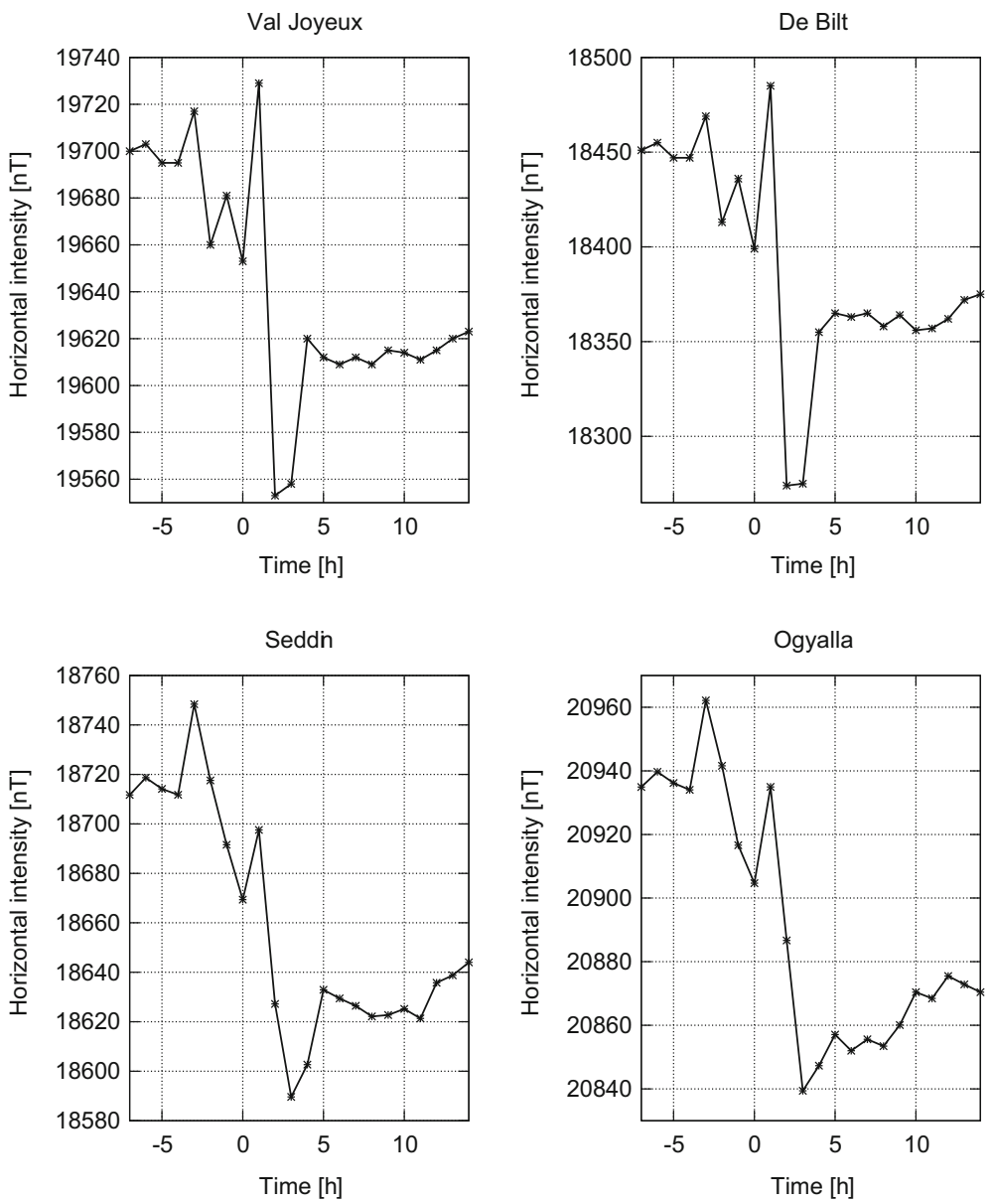

Fig. 2 One-hour means of horizontal component related to the magnetic storm of March 8, 1918, recorded by European magnetic observatories. The value 0 on the time axis corresponds to midnight

\section{Conclusion}

This study analysed the magnetogram of the intense magnetic storm of March 8, 1918, recorded by observatory Ógyalla (present day Hurbanovo). It was found that that event consisted of a pronounced storm sudden commencement (ssc), two auroral substorms that merged together forming a forceful disturbance, and the main phase caused by the ring current.

The disturbance caused by the ring current was rather intense; however, it was not an extreme one. Therefore, the strange and spectacular phenomena that were observed by the public and referred by contemporary newspapers, such as effects in telegraph lines, were likely due to the substorms. The rapid changes of the horizontal intensity during these substorms might be crucial here. The pronounced ssc represented another rapid change of the geomagnetic field that could be relevant. 

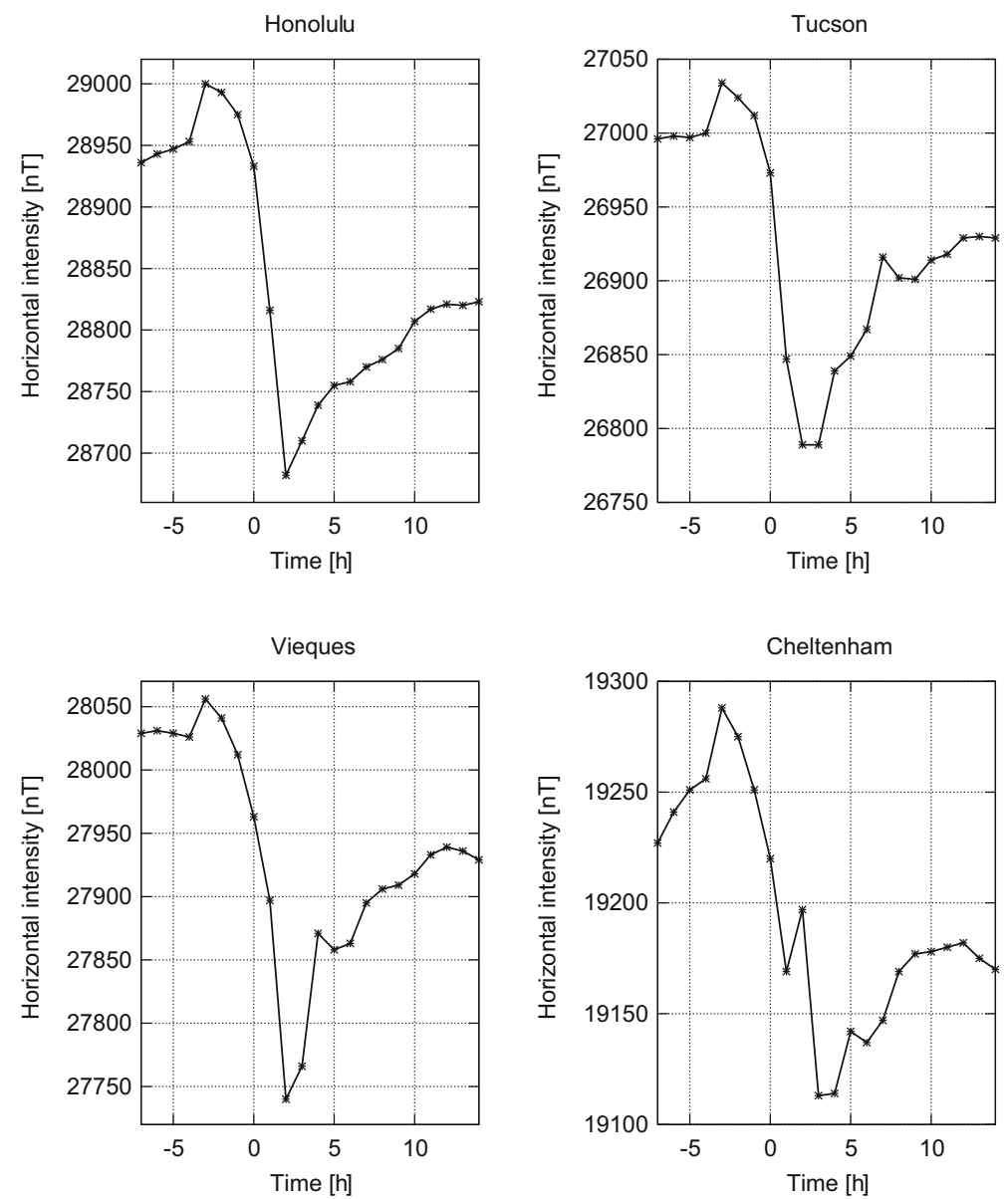

Fig. 3 One-hour means of horizontal component related to the magnetic storm of March 8, 1918, recorded by distant magnetic observatories. The value 0 on the time axis corresponds to midnight

It is generally accepted that substorms are caused by electric currents flowing at high latitudes and an ssc is related to enhanced magnetopause currents. The presented analysis thus supports the conclusion of Cid et al. (2015), who showed that the key feature of extreme magnetic disturbances at mid latitudes do not need to be directly connected with the ring current.

It would be interesting to know if there are any contemporary reports, such as newspaper articles, that informed about manifestations of the event of March 1918 in Central Europe. These might be auroras, which were probably visible at unusually low latitudes, weird behaviour of telegraphic lines, and so forth. Analyses of this kind of historical documents could eventually lead to better understanding of the risks associated with space weather.

Acknowledgments This work was supported in part by VEGA Grants Nos. 2/0030/14 and 2/0115/16 of the Scientific Grant Agency of the Ministry of Education of Slovak Republic and the Slovak Academy of Sciences, by the Slovak Research and Development Agency under the Contract No. APVV-0662-12. I 
would like to show my gratitude also to the institutions who provided the data that I used in this study, namely: the British Geological Survey for publishing the historical UK magnetic observatory magnetograms, the Ebre Observatory, Spain, for housing the International Service on Rapid Magnetic Variations, and the World Data Center for Geomagnetism, Kyoto, which is operated by Data Analysis Center for Geomagnetism and Space Magnetism, Graduate School of Science, Kyoto University, Japan, for collecting the 1-h geomagnetic data. Finally, I gratefully acknowledge the helpful comments of the anonymous referees.

\section{References}

Cid C, Saiz E, Guerrero A, Palacios J, Cerrato Y (2015) A Carrington-like geomagnetic storm observed in the 21st century. J Space Weather Space Clim 5:A16. doi:10.1051/swsc/2015017

Cliver EW, Dietrich WF (2013) The 1859 space weather event revisited: limits of extreme activity. J Space Weather Space Clim 3:A31. doi:10.1051/swsc/2013053

Ochabová P, Ochaba S (1977) The origin and development of the geomagnetic observatory in Hurbanovo. Contrib Geophys Inst Slovak Acad Sci 7:13-30

Odenwald S (2016) Space weather. http://www.solarstorms.org/SrefStorms.html. Accessed 14 Mar 2016

Prigancová A, Vörös Z (2001) On 100-year history of the Hurbanovo geomagnetic observatory. Contrib Geophys Geod 31(1):1-241

Saiz E, Guerrero A, Cid C, Palacios J, Cerrato Y (2016) Searching for Carrington-like events and their signatures and triggers. J Space Weather Space Clim 6:A6. doi:10.1051/swsc/2016001

Tsurutani BT, Gonzalez WD, Lakhina GS, Alex S (2003) The extreme magnetic storm of 1-2 September 1859. J Geophys Res 108(A7):1268. doi:10.1029/2002JA009504 